\title{
Variações nas dimensões do cristalino fummano de acordo com a idade
}

Variation of fuman lens dimensions according to age

\begin{tabular}{lcc} 
Mauro & \multicolumn{2}{c}{ Waiswol $^{1}$} \\
José & Wilson & Cursino $^{2}$ \\
Ralphł & Cohen $^{3}$
\end{tabular}

${ }^{1}$ Pós-graduando em Nível de Doutorado na Faculdade de Medicina da Universidade de São Paulo, Chefe da Seção de Catarata do Departamento de Oftalmologia da Santa Casa de São Paulo.

${ }^{2}$ Assistente Voluntário do Departamento de Oftalmologia da Santa Casa de São Paulo.

3 Professor Adjunto da Faculdade de Ciências Médicas da Santa Casa de São Paulo.

Endereço para correspondência: Av. Angélica, 916 - cj. 903 - São Paulo (SP) CEP 01228-000. E-mail: waiswol (@)yahoo.com

\section{RESUMO}

Objetivo: Demonstrar eventual relação entre a idade e o aumento da espessura e diâmetro do cristalino humano. Métodos: Diâmetro e espessura de 108 cristalinos removidos de olhos doados para transplante de córnea foram medidos com uso de paquímetro e microscopia estereoscópica. Resultados: As medidas médias obtidas com os cristalinos avaliados foram de $3,73 \mathrm{~mm}$ de espessura com desviopadrão de $0,73 \mathrm{~mm}$ e de $8,96 \mathrm{~mm}$ de diâmetro com desvio-padrão de 0,41 $\mathrm{mm}$. Sessenta e três cristalinos $(58,3 \%)$ provinham de indivíduos do sexo feminino e $45(41,7 \%)$, do sexo masculino, não havendo diferenças significativas entre os sexos relativamente à sua idade e à espessura e diâmetro dos cristalinos. Foi encontrada correlação significativa e diretamente proporcional entre a idade dos indivíduos e a espessura dos cristalinos, que mostrou aumento médio de 54,86\% entre os dez e os 90 anos de idade. Esta correlação não foi observada com relação ao diâmetro dos cristalinos. Conclusões: Ocorre aumento da espessura do cristalino em ambos os sexos e em todos os grupos etários estudados.

Descritores: Cristalino/crescimento e desenvolvimento; Fatores etários; Humano

O desenvolvimento pós-natal do cristalino humano não segue o mesmo padrão de desenvolvimento de outras estruturas do sistema nervoso central ou a ele associadas. Enquanto nos primeiros anos de vida o crescimento do cristalino parece seguir um padrão que é comum à maior parte das estruturas tanto do sistema nervoso central como do periférico, com o correr dos anos este crescimento continua em taxas estáveis e progressivas, ao passo que as demais estruturas chegam às suas dimensões finais ou muito próximas às finais pelo final da segunda década de vida ${ }^{(1)}$.

Curiosamente, o padrão de crescimento do cristalino humano também difere do de outras espécies animais, como ocorre com a vaca, o coelho e o rato, que apresentam um platô no crescimento do cristalino muito antes do curso da vida estar completo(2).

Os primeiros estudos sobre o crescimento do cristalino humano datam já de três séculos atrás ${ }^{(3-4)}$, e parece que a preocupação destes pesquisadores era entender as implicações do crescimento contínuo do cristalino sobre a ocorrência de glaucoma de ângulo fechado bem como os seus efeitos sobre a sua posição e sobre as constantes ópticas do olho ${ }^{(5-6)}$.

Diversos autores estudaram as dimensões do cristalino em condições 
como miopia, hipermetropia e emetropia, esperando encontrar alguma correlação com a refração ocular, o que, todavia, nunca foi comprovado ${ }^{(7)}$.

Já foi relatada uma série de estudos sobre as alterações em massa e densidade específica, volume, espessura e diâmetro do cristalino, todavia com resultados nem sempre concordantes no que se refere a estas alterações em função da idade. Neste sentido, Bron e Brown ${ }^{(7)}$ propuseram uma divisão didática para os períodos de crescimento do cristalino humano, a saber: período pré-natal; período pós-natal precoce, que abrange do nascimento aos 20 anos de idade; e o período pósnatal tardio, considerado a partir dos 20 anos de idade.

Com relação à massa do cristalino, há crescimento bastante acelerado durante a vida intra-uterina ${ }^{(1,8-9)}$, e consideravelmente menor na fase pós-natal. Crescimento linear na ordem de 1,32 mg/ano foi observado em grupos etários de 15 a 85 anos $^{(10-11)}$, sendo que o maior aumento de peso do cristalino ocorre nos primeiros 15 anos de vida, e de forma mais acelerada no primeiro ano de vida ${ }^{(1)}$. O ganho de peso após o final da primeira década de vida mostrou-se intimamente proporcional à idade ${ }^{(1)}$, diminuindo, porém, consideravelmente nos últimos anos de $\operatorname{vida}^{(11-12)}$.

Há pouca informação na literatura sobre o crescimento do volume do cristalino nas duas primeiras décadas de vida; a partir do final da segunda década, porém, parece haver um aumento estável no volume do cristalino, enquanto a gravidade específica mantém-se constante ${ }^{(1,4)}$. Alguns autores relataram frações relativamente fixas quanto ao peso líquido do cristalino, em amostras cujas idades oscilaram de 2,5 a 89 $\operatorname{anos}^{(4,11,13)}$; outros estudos, porém, sugerem evidências de um aumento contínuo e gradativo na gravidade específica após o nascimento, indicando desidratação pequena, mas estável, de todo o cristalino, levando à afirmação de que, da vida fetal à vida adulta, há redução do volume ocupado pelo cristalino no globo ocular ${ }^{(1,9,14-15)}$.

Alguns autores relataram taxa média de aumento da espessura do cristalino durante a vida intra-uterina na ordem de $7,6 \mathrm{~mm} /$ ano ${ }^{(16-17)}$. Contudo, poucos são os relatos sobre o crescimento da espessura do cristalino nas duas primeiras décadas de vida, e os resultados são conflitantes. Estudo ultra-sônico com 926 crianças, do nascimento à puberdade, mostrou que a espessura média do cristalino decresce cerca de $0,3 \mathrm{~mm}$ no primeiro ano de vida e outros $0,2 \mathrm{~mm}$ até os oito a dez anos de idade $^{(18)}$. Também Manzitti (citado Bron e Brown ${ }^{(7)}$ ) observou decréscimo na espessura do cristalino ao longo das duas primeiras décadas de vida. Outros estudos, todavia, evidenciaram achados bastante diferentes, segundo os quais há um aumento médio de espessura de $9 \mathrm{~mm}$ /ano neste mesmo período da vida ${ }^{(19-21)}$.

Quanto ao diâmetro do cristalino, já se observou que esta medida é de aproximadamente $6,3 \mathrm{~mm}$ ao nascimento, atingindo $9 \mathrm{~mm}$ ao final da segunda década de vida, a partir de quando este diâmetro continua crescendo, ainda que em taxas mais sutis $^{(4,17,22-24)}$.
Neste estudo objetivamos observar o crescimento da espessura e diâmetro do cristalino em função da idade, contribuindo com achados mais recentes sobre o tema, já que a literatura a ele pertinente é relativamente antiga, e especificamente com dados de amostra composta de indivíduos brasileiros.

\section{MÉTODOS}

Estudaram-se cristalinos humanos removidos de olhos doados para o Banco de Olhos da Santa Casa de São Paulo e para o Banco de Olhos de Sorocaba, no período de julho de 1999 a maio de 2000, excluindo-se aqueles malformados ou rotos. Os 108 cristalinos incluídos no estudo apresentavam idade mínima de 13 anos e idade máxima de 87 anos e foram distribuídos de acordo com a idade em intervalos de cinco anos, procurando representar com veracidade cada intervalo analisado. Os cristalinos foram preservados em solução de formaldeído a $10 \%$. Foram excluídos cristalinos que apresentavam ruptura capsular e deformidades. Para as medições, cada cristalino foi colocado sobre papel filtro para remoção do filme do fixador e secagem. Com o uso de paquímetro colocado abaixo do microscópio estereoscópico marca American Optical, modelo 570/07 TO 4.2X, com objetiva de aumento de dez vezes, foram realizadas três medidas dos diâmetros (de equador a equador) e espessuras (antero-posterior) de cada cristalino.

A partir das três medidas da espessura e das três medidas do diâmetro realizadas em cada cristalino, foram calculadas respectivas médias aritméticas e desvios-padrão.

Os dados assim obtidos foram estatisticamente analisados para um nível de probabilidade de $5 \%(\mathrm{p} \leq 0,05)$.

Para eventual identificação de diferenças entre os sexos dos indivíduos dos quais provieram os cristalinos no que se refere às suas idades e ao diâmetro e espessura dos cristalinos, empregou-se o Teste t de Student. A correlação paramétrica de Pearson foi empregada para a análise de eventuais associações entre as variáveis estudadas.

Para a compreensão das correlações encontradas especificamente em função da idade, utilizaram-se, ainda, cálculos de regressão linear e intervalo de confiança individual.

\section{RESULTADOS}

As médias aritméticas e desvios-padrão para cada uma das três medidas realizadas para espessura e diâmetro dos 108 cristalinos avaliados encontram-se na tabela 1 .

Sessenta e três $(58,3 \%)$ cristalinos pertenciam a indivíduos do sexo feminino e $45(41,7 \%)$, do masculino. A média geral de idade dos indivíduos cujos cristalinos foram estudados foi de $54,57+17,22$ anos.

Estes cristalinos foram divididos em dois grupos, de acordo com o sexo dos indivíduos dos quais provinham, e assim 


\begin{tabular}{|lcccc|}
\hline $\begin{array}{l}\text { Tabela 1. Médias aritméticas e desvios-padrão (em milímetros) } \\
\text { para as três medidas de espessura e diâmetro de } \\
\text { humanos }\end{array}$ \\
$\begin{array}{l}\text { Medidas } \\
\text { Meristalinos }\end{array}$ \\
$\begin{array}{l}\text { Média } \\
\text { Espessura }\end{array}$ & $\begin{array}{c}\text { Diâmetro } \\
\text { Desvio-padrão }\end{array}$ & $\begin{array}{c}\text { Média } \\
\text { Desvio-padrão }\end{array}$ \\
Segunda medida & 3,72 & 0,72 & 8,95 & 0,42 \\
Terceira medida & 3,73 & 0,74 & 8,97 & 0,41 \\
Valores finais & 3,73 & 0,73 & 8,96 & 0,41 \\
\hline
\end{tabular}

distribuídos de acordo com a média etária destes indivíduos (Tabela 2) e com os valores médios relativos à sua espessura $\mathrm{e}$ diâmetro (Tabelas 3 e 4). Deste modo, não se encontraram diferenças significativas entre os sexos com relação às idades dos indivíduos nem no que concerne aos valores médios de espessura e diâmetro dos cristalinos estudados.

A correlação paramétrica de Pearson entre idade e sexo dos indivíduos e valores médios de espessura e diâmetro dos cristalinos evidenciou coeficiente de correlação significativo e diretamente proporcional entre a idade dos indivíduos e a espessura dos cristalinos $(\mathrm{r}=0,54 ; \mathrm{p}=0,0001)$ bem como entre a espessura e o diâmetro dos cristalinos $(\mathrm{r}=0,23 ; \mathrm{p}=0,018)$.

Utilizando o modelo de regressão linear e colocando os valores médios de espessura dos cristalinos em função da idade dos indivíduos, observou-se que a espessura cresce com o correr da idade, evidenciando proporcionalidade direta entre as variáveis. A reta representativa dos valores médios da espessura dos cristalinos em função da idade dos indivíduos está ilustrada no gráfico 1.

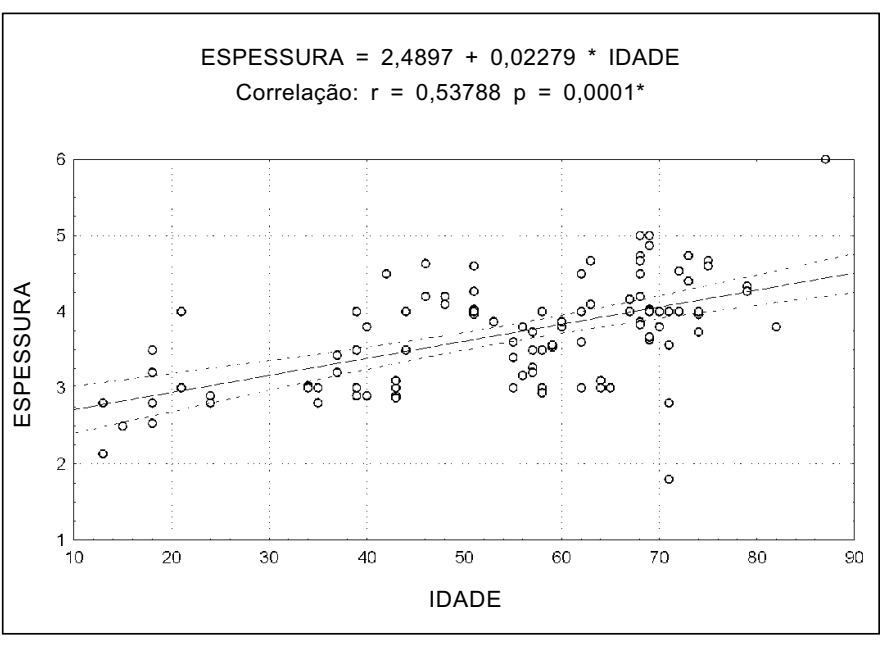

Gráfico 1 - Reta representativa dos valores médios da espessura (em milímetros) dos cristalinos em função da idade (em anos) dos indivíduos

O intervalo de confiança da reta de regressão para valores individuais foi calculado com a seguinte fórmula:

$$
Y^{\prime} \pm t_{(n-2)} \sqrt{s_{y \cdot x}^{2}\left[1+\frac{1}{n}+\frac{(X-\bar{X})^{2}}{\sum(X-\bar{X})^{2}}\right]}
$$

Onde:

$\mathrm{X}=$ cada um dos valores da idade

$+Y^{\prime}=$ limites mínimo e máximo dos valores de espessura para cada valor de idade

$\mathrm{S}_{\mathrm{y} . \mathrm{x}}=$ erro padrão da estimativa

Tabela 2. Médias aritméticas, desvios-padrão e valores mínimos e máximos relativos à idade (em anos) dos indivíduos distribuídos de acordo com o sexo

\begin{tabular}{lccccccc} 
Sexo & N & Média & Desvio-padrão & Valor mínimo & Valor máximo & Teste T & Probabilidade \\
Feminino & 63 & 54,60 & 17,99 & 13,00 & 82,00 & \\
Masculino & 45 & 54,53 & 16,28 & 15,00 & 87,00 & 0,02 & $\mathrm{p}=0,984$ \\
Valores finais & 108 & 54,57 & 17,22 & 13,00 & 87,00 & & \\
\hline
\end{tabular}

Tabela 3. Médias aritméticas, desvios-padrão e valores mínimos e máximos relativos à espessura (em milímetros) dos cristalinos, distribuídos de acordo com o sexo dos indivíduos dos quais provieram

\begin{tabular}{|lccccccc} 
Sexo & N & Média & Desvio-padrão & Valor mínimo & Valor máximo & Teste T & Probabilidade \\
Feminino & 63 & 3,80 & 0,68 & 2,13 & 6,07 & 1,12 & $p=0,265$ \\
Masculino & 45 & 3,64 & 0,80 & 1,80 & 6,00 & 6,07 & \\
\hline Valores finais & 108 & 3,73 & 0,73 & 1,80 & & \\
\hline
\end{tabular}

Tabela 4. Médias aritméticas, desvios-padrão e valores mínimos e máximos relativos ao diâmetro (em milímetros) dos cristalinos, distribuídos de acordo com o sexo dos indivíduos dos quais provieram

\begin{tabular}{lccccccc} 
Sexo & $\mathbf{N}$ & Média & Desvio-padrão & Valor mínimo & Valor máximo & Teste T & Probabilidade \\
Feminino & 63 & 8,92 & 0,29 & 8,00 & 9,50 & 1,26 & $\mathrm{p}=0,211$ \\
Masculino & 45 & 9,02 & 0,53 & 6,03 & 10,00 & 1 \\
Valores finais & 108 & 8,96 & 0,41 & 6,03 & 10,00 & & \\
\hline
\end{tabular}


O gráfico 2 mostra, assim, a reta de regressão dos valores de espessura em função da idade, o intervalo de confiança da média e o intervalo de confiança individual formado pelas margens externas, ligeiramente bicôncavas. O espaço compreendido entre as margens externas inclui $95 \%$ dos casos analisados, tendo em vista o nível de probabilidade adotado para este estudo $(p \leq 0,05)$, o que permitiu o cálculo dos valores médios, mínimos e máximos da espessura dos cristalinos em função da idade dos indivíduos, com o emprego da referida fórmula.

Os valores mínimos, médios e máximos de espessura dos cristalinos foram distribuídos de acordo com as idades dos indivíduos, relacionados em intervalos de cinco anos, numa escala crescentes de dez a 90 anos (Tabela 5), obtendo-se, assim, percentual de $54,86 \%$ de aumento da espessura do cristalino nesta escala etária, o que equivale a um aumento de $6,86 \%$ em cada década de vida ou de $0,686 \%$ em cada ano de vida.

\section{DISCUSSÃO}

Anatomicamente, o cristalino é formado por um envelope externo, que é a sua cápsula, uma monocamada celular anterior, epitélio e fibras, que formam a maior parte do cristalino, arranjadas em gerações concêntricas.

A cápsula do cristalino, como qualquer outra lâmina basal, funciona como reservatório para os fatores de crescimento, permitindo sua ação prolongada em relação a órgãos-alvo específicos. Assim, ela é remodelada durante toda a vida útil do cristalino, em resposta ao aumento contínuo do volume do cristalino, que é acompanhado pelo aumento do seu diâmetro devido, por sua vez, provavelmente ao aumento no número e/ ou na espessura das fibras.

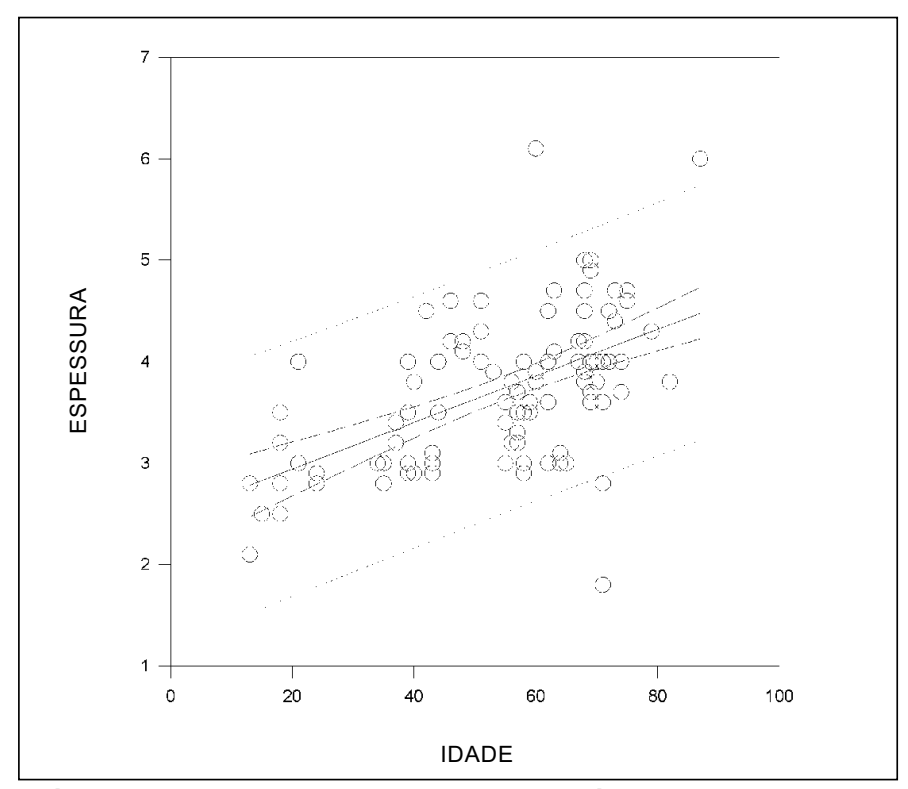

Gráfico 2 - Reta de regressão dos valores médios de espessura (em milímetros) dos cristalinos em função da idade (em anos) dos pacientes

\begin{tabular}{|c|c|c|c|}
\hline \multirow[t]{2}{*}{ Idade (em anos) } & \multicolumn{3}{|c|}{ Espessura (em milímetros) } \\
\hline & Mínima & Média & Máxima \\
\hline 10 & 1,6 & 2,9 & 4,2 \\
\hline 15 & 1,7 & 3,0 & 4,3 \\
\hline 20 & 1,8 & 3,1 & 4,4 \\
\hline 25 & 1,9 & 3,2 & 4,5 \\
\hline 30 & 2,0 & 3,3 & 4,5 \\
\hline 35 & 2,1 & 3,4 & 4,6 \\
\hline 40 & 2,2 & 3,5 & 4,7 \\
\hline 45 & 2,3 & 3,6 & 4,8 \\
\hline 50 & 2,4 & 3,7 & 4,9 \\
\hline 55 & 2,5 & 3,8 & 5,0 \\
\hline 60 & 2,6 & 3,9 & 5,1 \\
\hline 65 & 2,7 & 4,0 & 5,2 \\
\hline 70 & 2,8 & 4,1 & 5,3 \\
\hline 75 & 2,9 & 4,2 & 5,4 \\
\hline 80 & 3,0 & 4,3 & 5,5 \\
\hline 85 & 3,1 & 4,4 & 5,6 \\
\hline 90 & 3,2 & 4,5 & 5,7 \\
\hline
\end{tabular}

Ao nascimento, o cristalino apresenta forma esférica, sendo porém mais longo o seu plano equatorial. Nas primeiras duas décadas pós-natais, a expansão do plano sagital cessa, continuando apenas a expansão do plano equatorial. Nestas primeiras décadas, também ocorre a compactação nuclear que, ao terminar, possibilita o reinício da expansão sagital.

Muitos estudos já foram realizados no sentido de se compreender melhor a maneira como ocorre o crescimento do cristalino no curso da vida, a uma grande parte dos quais, todavia, se atribui achados fragmentados em decorrência de erros metodológicos ${ }^{(17)}$.

Nosso estudo, realizado com 108 olhos doados para transplante de córnea e provenientes de indivíduos de ambos os sexos e com idades entre 13 e 87 anos, evidenciou medidas médias de espessura e diâmetro do cristalino de $3,73 \mathrm{~mm}$ e $8,96 \mathrm{~mm}$, respectivamente, valores esses equivalentes aos relatados pela literatura em amostras sócio-culturais diferentes da nossa ${ }^{(1,4,7,10-11,14)}$.

Embora haja relatos de que o cristalino das mulheres tende a ser discretamente menor do que o dos homens ${ }^{(25)}$, não encontramos quaisquer diferenças importantes no que se refere à espessura e ao diâmetro do cristalino de olhos provenientes de 63 mulheres e 45 homens.

Dentre os problemas metodológicos citados por Weale ${ }^{(17)}$ com relação aos estudos que se propuseram compreender o crescimento do cristalino, encontra-se a fixação dos olhos em formaldeído anteriormente à sua medição, uma vez que pode levar tanto a um inchaço como, mais comumente, a um encolhimento desta estrutura ocular, resultando em medidas subestimadas, comparativamente às medidas do cristalino in vivo. Este fenômeno, inclusive, parece influenciar mais estruturas oculares de crianças e jovens, acarretando efeitos menores em cristalinos de indivíduos mais velhos ${ }^{(25)}$. 
Todavia, embora em nossas observações as medidas do diâmetro e espessura do cristalino possam estar ligeiramente alteradas, uma vez que os olhos estudados foram preservados em formaldeído por um tempo médio de quatro dias, este bias metodológico não influencia os nossos achados, pois, provavelmente, a mesma proporcionalidade direta entre aumento e avanço da idade fica mantida para a compreensão do desenvolvimento do cristalino in vivo.

De fato, nossos resultados apontaram que o aumento da espessura do cristalino acompanha o avanço da idade em proporções diretas. Igualmente, o aumento do diâmetro do cristalino mostrou correlação significativa com o aumento de sua espessura. E, embora a correlação entre idade e diâmetro não tenha se mostrado estatisticamente significativa, este resultado evidenciou forte tendência a uma proporcionalidade direta, no caso de uma amostra maior.

Neste sentido, não podemos concordar com autores que, no passado, relataram diminuição nas medidas da espessura do cristalino na primeira ou mesmo nas duas primeiras décadas de vida ${ }^{(18)}$. Por outro lado, corroboramos os achados de autores que observaram aumento na espessura do cristalino nas duas primeiras décadas de vida ${ }^{(20)}$.

$\mathrm{O}$ aumento nas medidas do cristalino parece ser devido à ação da tensão zonular sobre o cristalino e ao aumento desta tensão após o nascimento, que resulta, por sua vez, do aumento contínuo do tamanho do globo ocular ${ }^{(17)}$. Parece que o maior crescimento sagital contínuo do globo ocular ocorre nos três a cinco primeiros anos de vida, cessando, finalmente, por volta do final da primeira década de vida, enquanto o crescimento gradual da espessura do cristalino é mais vagaroso por tempo consideravelmente maior. A expansão sagital do cristalino é, então, reacelerada, a partir dos 20 anos até perto $\operatorname{dos} 80 \operatorname{anos}^{(17)}$.

Destes estudos, infere-se que haveria um platô no crescimento da espessura do cristalino nas primeiras duas décadas de vida; mas, nenhuma explicação para este platô foi ainda aceita $^{(7)}$, uma vez que não existe informação suficiente sobre o que de fato acontece em nível celular com relação ao volume do cristalino neste período da vida.

Não podemos afirmar a taxa de aumento do diâmetro e espessura do cristalino antes do início da segunda década de vida, uma vez que os olhos que estudamos eram de indivíduos com idade mínima de 13 anos. Mas nos ficou evidente que, a partir dos dez anos de idade, a espessura do cristalino cresce gradualmente, na ordem de $0,686 \%$ ao ano, com o passar da vida, pelo menos até os 90 anos. Com isso, a hipótese levantada segundo a qual há um platô no crescimento da espessura do cristalino nos primeiros vinte anos de vida nos parece digna de novas refutações, uma vez que observamos este crescimento entre os dez e 20 anos de idade nas mesmas proporções que nas décadas subseqüentes.

O cristalino apesar de já ter sido estudado ao longo do tempo, voltou a ter importância prática, quando mecanismos de acomodação são discutidos, cirurgias para presbiopia são propostas e lentes acomodativas são desenvolvidas.

\section{CONCLUSÃO}

Este estudo permite concluir que há aumento progressivo da espessura do cristalino ao longo da vida, em proporção direta com o avanço da idade, independentemente do sexo. Entre os dez e os 90 anos de idade, este aumento atinge taxa de $54,86 \%$.

S UMMARY

Purpose: To determine an eventual relationship between human lens thickness and age. Methods: Lens thickness and diameter were assessed by pachymetry and stereoscopic microscopy in 108 eyes donated for corneal graft. Results: Mean lens thickness was $3.73 \mathrm{~mm}$ with standard deviation of $0.73 \mathrm{~mm}$ and mean diameter was $8,96 \mathrm{~mm}$ with standard deviation of $0.41 \mathrm{~mm}$. Sixty-three lenses $(58.3 \%)$ were from female subjects and forty-five (41.7\%) were from male subjects. There was no significant difference of lens diameter and thickness between genders. Age and lens thickness presented a significant direct correlation with a $54.86 \mathrm{~mm}$ mean increase between ages ten and ninety. This correlation was not observed in relation to the lens diameter. Conclusion: With age, lens thickness presented a significant increase among all studied age groups.

Keywords: Lens; crystalline/growth \& development; Age factors; Human

\section{REFERE N C IAS}

1. Scammon RE, Hesdorffer MB. Growth in mass and volume of the human lens in postnatal life. Arch Ophthalmol 1937;17:104-12.

2. Van Heyningen R. Experimental studies on cataract. Invest Ophthalmol Vis Sci 1976;15:685-97.

3. Du Petit FP. Memoire sur plusiers decouvertes faites dans les yeux d l'homme, des animaux a 4 pieds, des oiseaux et des poissons. Mem Acad Royal Sci 1723;1:1.

4. Smith P. The growth of the crystalline lens. Br Med J 1883;1:112.

5. Smith P. Glaucoma problems. Ophthalmic Rev 1911;30:31.

6. Smith P. Glaucoma problems. Ophthalmic Rev 1912;31:129-89.

7. Bron AJ, Brown NAP. Growth of the human lens: the lens as a clock. In: Cotlier E, Lambert S, Taylor D, editors. Congenital cataracts. Austin: RG Landes Company; 1994. p.203-31.

8. Ehlers N, Matthiessen ME, Andersen H. The prenatal growth of the human eye. Acta Ophthalmol (Copenh)1968,46:329-49.

9. Bours J, Fudisch HJ. Human fetal lens: wet and dry weight with increasing gestational age. Ophthalmic Res 1986;18:363-8.

10. Spencer RP. Change in weight of the human lens with age. Ann Ophthalmol 1976;8:440-1.

11. Nordmann J, Fink H, Hockwin O. Die Wachstumskurve der manschlichen Linse. Arch Klin Exp Ophthalmol 1994;191:165.

12. Rodriguez-Caballero ML, Gerhard JP, Nordmann J. L'épaisseur corticale du cristallin humain. Doc Ophthalmol 1973;35:287-95.

13. Fisher RF, Pettet BE. Presbyopia and the water content of the human crystalline lens. J Physiol 1973;234:443-7.

14. Bours J, Fodisch HJ. Human fetal lens: wet and dry weight with increasing gestational age. Ophthalmic Res 1986;18:363-8.

15. Clapp CA. A communication on infant lenses and their solids. Arch Ophthalmol 1913;42:618. 
16. Collins ET. Lectures on the anatomy and pathology of the eye. Lancet 1894;2:1329-37. apud Bron AJ, Brown NAP. Growth of the human lens: the lens as a clock. In: Cotlier E, Lambert S, Taylor D, editors. Congenital cataracts. Austin: RG Landes Company; 1994. p.203-31.

17. Weale RA. Biography of the eye: development, growth, age. London: HK Lewis; 1982. p.1.

18. Larsen JS. The sagittal growth of the eye. Acta Ophthalmol (Copenh) 1971; 49:427-40.

19. Forbes JE, Holden R, Harris M, Brown NP, Bron AJ. Growth of the crystalline lens in childhood. Oxford: Oxford Congress; 1992.

20. Forbes JE, Holden R, Harris M, Brown NAP, Bron AJ. Growth of the human crystalline lens in childhood [abstract 578]. Exp Eye Res 1992;55:172.
21. Dub B. Beitraege zur Kenntnis der Catarata zonularis. 1891 apud Bron AJ, Brown NAP. Growth of the human lens: the lens as a clock. In: Cotlier E, Lambert S, Taylor D, editors. Congenital cataracts. Austin: RG Landes Company; 1994. p.203-31.

22. Pierscionek BK. Prebyopia: the effect of refractive index. Clin Exp Optom 1990;73:23-30.

23. Scammon RE, Wilmer HA. Growth of the components of the human eyeball. II. Arch Ophthalmol 1950;43:620-37.

24. Fisher RF. The elastic constants of the human lens capsule. J Physiol 1969; 201:1-19.

25. Pau H. Lisenquellung im physiologischen Millieu. Arch Ophthalmol (Berlim) 1951;151:352.

\title{
VII CONGRESSO INTERNACIONAL de catarata e cIRURGia REFRATIVA
}

\author{
6 a 9 de Abril de 2002
}

\section{Hotel Transamérica - São Paulo - SP}

Organização: Sociedade Brasileira de Catarata e Implantes Intraoculares

e

Sociedade Brasileira de Cirurgia Refrativa

Informações: JDE Comunicação e Eventos

Alameda Santos, 1.343 - Cj. 304

Cep 01419-001 - São Paulo - SP

Telf.: (1 1) 287-8109 / 287-9699 - Fax: 288-8157

E-mail: jdecomev@uol.com.br 